\title{
TOWARDS A VIRTUAL ORGANIZATION: THE RAPID PROTOTYPING PORTUGUESE NATIONAL NETWORK
}

\author{
Pedro A. C. Sousa \\ pas@uninova.pt \\ João P. Pimentão \\ pim@uninova.pt \\ Pedro M. N. Maló \\ pmm@uninova.pt \\ Ricardo Gonçalves \\ rg@uninova.pt \\ Adolfo Steiger-Garção \\ asg@uninova.pt \\ UNINOVA - Instituto de Desenvolvimento de Novas Tecnologias, \\ Universidade Nova de Lisboa Faculdade de Ciências e Tecnologia-PORTUGAL
}

\begin{abstract}
The integration of state-of-the-art Information Technology (IT) into the rapid prototyping (RP') industry has not yet achieved a satisfactory degree of implementation. Most established developments are focused on the Computer-Aided Design (CAD), drafting, and automation of certain pre and post fabrication processes. This paper outlines the architecture, designed under UNINOVA's co-ordination, for the overall incorporation of information technologies (IT), in the rapid prototyping industry, in order to create the Infrastructures for the RP Portuguese National Network, which can be seen as a virtual organization.

RNPR's virtual organization provides a global service as the outcome of all partners' composite skills. Such a virtual composite organization can provide a better service, either in terms of quality, diversity, applicability or time constraints.
\end{abstract}

\section{INTRODUCTION}

The need of cost and development time reduction, combined with the demand to increase the ability to launch high quality innovative products into the market led to the creation of Rapid Prototyping (RP) technologies and to the development of concepts such as Rapid Tooling (RT), Rapid Manufacturing (RM) and Reverse Engineering (RE). In a broad sense, these technologies have been successfully used to meet those challenges. Nowadays, these technologies are considered to be powerful tools in the development of products in wide-ranging industrial fields, 
forcing the adaptation of the pre-production phase of the traditional product development life cycle towards its integration.

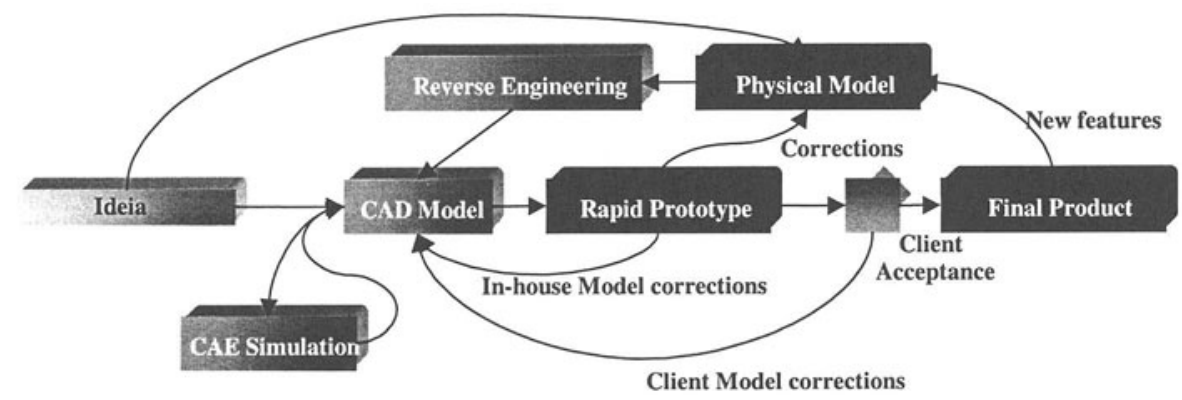

Figure 1 - Product life-cycle pre-production phase towards new technologies integration

Although the introduction of these technologies in the product development cycle implies extra direct costs and time spent, it is now also accepted that the total time and cost development can be drastically reduced.

\subsection{Rapid prototyping \& Reverse engineering}

Rapid Prototyping (RP) consists of various manufacturing processes, by which a solid physical model of a part is produced directly from a 3D CAD model. An essential pre-requisite is the computer representation of the part, obtained from a geometrical modelling system - like a CAD system - a 3D scanner or a computerised topography generator.

In contrast with traditional prototyping, which is usually slow and expensive, Rapid Prototyping techniques allow the fast production of physical pre-products with the important benefit of reducing the Time To Market factor. Through the use of such techniques, prototypes can be built, needing the skills of individual craftsmen for no more than just the finishing of the part. Furthermore, RP eliminates inevitable errors, which usually appear when a model-maker interprets a set of drawings (Dutta, 1992).

Another obvious advantage is that the same data used to create the prototype can be used to go directly from prototype to production, eliminating effort duplication, thus saving resources and preventing further errors. The base of all Rapid Prototyping processes is the $3 \mathrm{D}$ CAD product data slicing into thin $(0.01 \mathrm{~cm})$ crosssectional planes, by a computer, which are used to build the part layer-by-layer (Donahue, 1991).

Figure 2 presents several prototypes produced inside the RNPR organization using different technologies. 

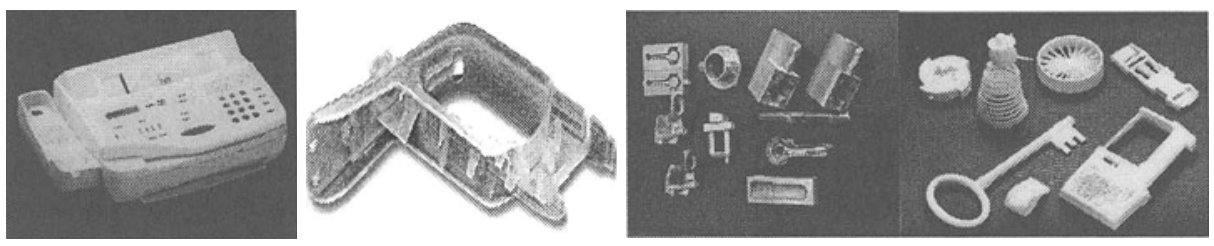

Figure 2 - Prototype examples built within the RNPR consortium

Another important characteristic of all RP techniques is the possibility of exercising the so-called reverse engineering (RE), i.e., obtaining the CAD model from the physical model. RE enables the object dimensional validation using an automatic process based on digitalisation followed by CAD geometrical representation creation. The different techniques allow an average accuracy of the hundredth of millimetres.

\subsection{Information technologies}

As mentioned earlier, speed is one of the most distinguishing features of RP technology when compared to conventional methods. In fact, in many cases, the use of RP can only be justified if the part can be obtained quickly. Quite often, the limiting factor is the time spent preparing the data and transferring it to the RP machines. When the data is considered valid and deployed into the RP machine facilities, manufacturing time is well known and relatively fast. In order to speed up the overall process, the existence of good communication systems is crucial to overcome the implicit drawback in the manipulation of average RP files, usually megabytes sized. Given this, and considering the specific scope of RP's Virtual Organisation (VO) an high communication throughput is essential, besides the usual relevant VO requirements: availability, quality, information security/privacy and integrity, authentication, date and time co-ordination, delivery report and receipt notification (Osório, 1999). Together with good communication infrastructures, the use of CAD systems, Database Management Systems (DBMS) and Expert Systems (ES) is important to manage and validate data, to support the identification of errors and to propose solutions, to check product conformance and production viability and to seek intelligent product development.

\subsection{RNPR Virtual Organization justification}

Although, with promising possibilities, RP's practical application is still very limited and confined to large manufacturers. At the top of list of major reasons for this situation it is possible to identify:

- Socio-Cultural reasons - The lack of knowledge of RP technologies, which is difficult to overcome due to the wide range of possible techniques;

- Economical reasons - The time consumed to fully dominate RP technologies and the prohibitive costs of each machine (more than one hundred thousand Euro);

- Vertical market embracing - Each technology has a defined sphere of applicability which reduces the possibility of its application to different types of products. The same manufacturers usually produce products, whose parts should be prototyped using different RP technologies. 
The reasons listed contribute for the lack of dissemination and application of RP technologies even in large companies, because of their difficult ROI (Return Of Investment) and exclude SME, to create a complete RP and RE environment.

Therefore, the outsourcing of RP and RE services is the most common approach to the minority of companies that use these techniques, but even in this cases, the information on the technological differences and separate offers, are barriers that are difficult to overcome.

The creation of a Virtual Organisation, that offers a wide range of technologies, each with its specific range of applicability, to different clients geographically spread becomes crucial. This VO provides a global service, as the outcome of all partners' composite skills, providing a better service, both in terms of quality, diversity, applicability and time constraints. The underlying concept of RNPR, is to enable each of the project partners to proclaim, to their own customers, their added value in the development of rapid prototypes, using every technology available in each of the consortium nodes. Each RNPR node then acts as a seller of its own valences and a reseller of the services provided by the RNPR. This results on an added set of services, which, potentially, can bring more customers. The fact that these customers may approach the node to request the services provided by the RNPR, may be a way of attracting them to the services that are the core competence of the node.

\section{RNPR - PORTUGUESE RAPID PROTOTYPING NETWORK}

The PEDIP II RNPR is a 4 years project, started in January 97. Its main objective is to create a Portuguese Network for Rapid Prototyping, supported by a set of RP and $\mathrm{RE}$ machines located country wide, and interconnected using the Internet. The total project budget is approximately seven million Euros.

The final objective is to have a completely integrated global customerproduction chain, where RP and RE are supplied, using the most common standards.

The project objectives are also underlying to conceptual product development support, not just at engineering design level, but also to assist the overcoming of major problems concerning the integration in the $\mathrm{CAD} / \mathrm{CAM}$ environments.

One of the main problems to be solved during this project is the integration of data between different heterogeneous applications, like CAD, ES, DBMS and RP machines. (Boissier, 1995) To help in this situation, data format conversion services were implemented, thus enabling data system heterogeneity exchange.

For prototype development, the project considered a relatively wide scope of RP techniques, namely SL (Stereo-lithography), LOM (Layer Object Model), 3-D printing HSM (High Speed Machining), SLS (Selective Laser Sintering) and DMLS (Direct Metal Laser Sintering), each with its specific range of applicability.

Traditional industries like furniture, foundry (production of moulds and cores), investment casting (production of wax models or moulds for wax models) or injection mould manufacturing (production of inserts or other components) were chosen to be the test bed of RNPR and the target beneficiaries. 


\section{INFORMATION TECHNOLOGY - RNPR'S BACKBONE}

The overall incorporation of information technologies (IT) in the rapid prototyping industry, in order to create the infrastructures for the RP Portuguese National Network, which can be seen as a virtual organisation, was designed under the co-ordination of the Research Institute UNINOVA.

An Internet-based architecture was designed to support the RNPR's virtual organization interface. This virtual interface provides a global service, from the RNPR client's perspective granted by a single entity, as the outcome of all partners' composite skills. The partners are geographically distributed throughout the country. During project execution, the Internet address of RNPR's portal is http://www.rnpr.net.

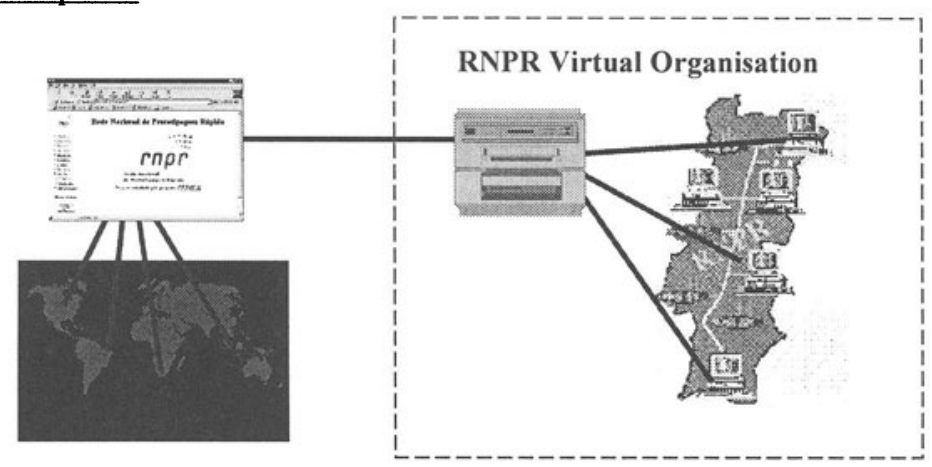

Figure 3 - RNPR Portal distributed access

The extensive use of Information Technology enabled an effortless integrated services' offer and contributed for a more flexible business process implementation, not only to the customer but also to the partners of the consortium, augment by a shared data and knowledge philosophy and communication infrastructures. For these purposes, an Internet based Information System was developed and installed, enabling data exchange support. This system is composed by a set of servers, which enable integrated services of WWW, FTP, file archive management, electronic Email, Email lists and videoconference. It supports the establishment of one-to-one and one-to-many communication channels between the RNPR clients and the consortium, as well as between the partners within the consortium, supporting geographically distributed collaborative work. The RNPR system also acts as a formation/educational system, since it stores technology description, samples, glossary of terms, and other related information.

In order to support the described services, distinct levels of security were enforced to ensure data confidentiality and integrity, an imperative in industrial environments. Therefore, it is possible to distinguish ownership at different levels, consortium partner data, consortium data, and general public access data. The data integrity is assured, even at the case of data transfer to the general public, since the connection can be made through a secure channel, based on a security system, for instance, using SSL (Secure Socket Layer) peer-to-peer protocol.

\subsection{RNPR's integrated services}

Two types of services were defined, based on the targeted user: the services for 
RNPR partners and those for the potential customers or RP technology newcomers.

\subsubsection{RNPR services for partners}

Each member of the consortium identifies itself to the RNPR portal using a login and a password, which enables the access to additional functionalities (e.g. maintenance facilities presented later on).

\section{The Decision Support System}

The mobility of human resources has demonstrated that the investment put in the training of a given technician (or team of technicians) may be wasted when the market shows the lack of highly skilled persons. Knowledge is one of the most precious assets of any given company and should be kept in house, at all cost.

In order to help achieve this goal, a Decision Support System (DSS) was designed to allow each of the technology holder's institutions, to maintain a database of developed projects. This database encompasses the whole process of prototype development, from the budget negotiation, to the phases of the prototype's production, keeping information on the resources and results of each phase, which, later on, permits report generation, such as production costs details. The DSS helps technology selection based on database search through similar projects, both in budget negotiation or production phases.

The system also allows a user at a given institution within the RNPR group to select a set of projects that may be made available to the consortium. The collecting of such data acts also as a repository of historical of each partner, in which data from projects - phases, duration, resources and others - is stored.

\section{The File Format Conversion Service}

The STL (Standard Triangulation Language) is a de facto standard in Rapid Prototyping, accepted by every RP machine (Figure 4). One of the problems with this format is that only a small number of CAD systems are able to "understand" it.
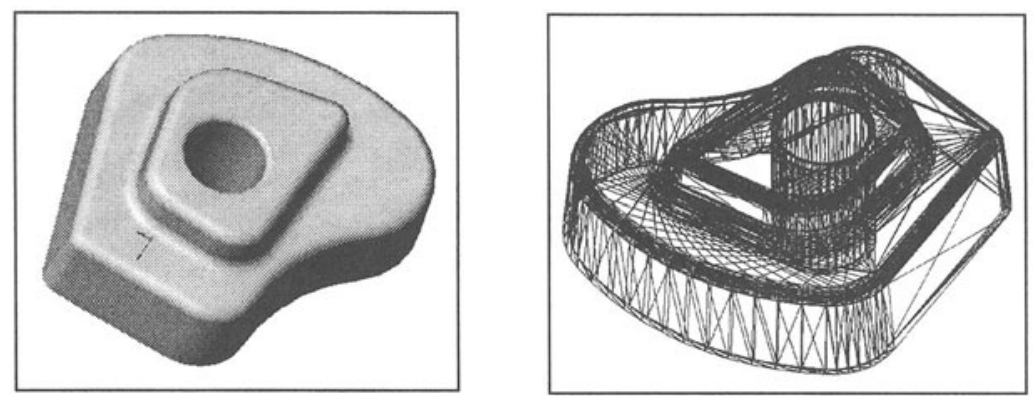

Figure 4 - Surface and it's RP description as a set of triangular plane facets

To help cope with this problem, a set of translators was created for some of the most commonly used formats. This service allows a user to submit a file in a given format (either binary or plain ASCII text STL) to the portal and to be able to retrieve the conversion results, in the selected format, later on.

Translations are available to DXF, OFF and VRML. The later is one of the most interesting results, since the output can be viewed with an Internet Browser using a public domain plug-in.

Some effort has been put in ISO to create a standard that would solve once and 
for all the CAD data conversion problem between CAD systems. ISO's proposal is ISO project 10303 STEP - Standard for the exchange of product data. UNINOVA has produced a converter that allows STL files to be converted into ISO's Application Protocol 214 that is readable by most of the professional CAD systems.

With the introduction of the STEP standard, it is expected that, in short term, a change from the current reality will be attained, converging to a single standardized format without information losses (IS0, 1997)(ISO, 1992).

\subsubsection{Services for the customer or RP newcomer}

The potential customer may benefit from some of the services that were designed for the consortium member, in the perspective of a user of such services, not being allowed maintenance facilities.

\section{The educational service}

An effort has been put into the development of a system that might help an ordinary user to understand the rapid prototyping cycle, to identify it's position regarding the use of this technology and determine which steps does it need to take, in order to use the technology. Also, this system would help to identify the set of the portal services that are available to accomplish some of the steps of the process.

The user is presented with a flowchart (Figure 5), where each node leads him to the detailed description of the related concept/process/technology.
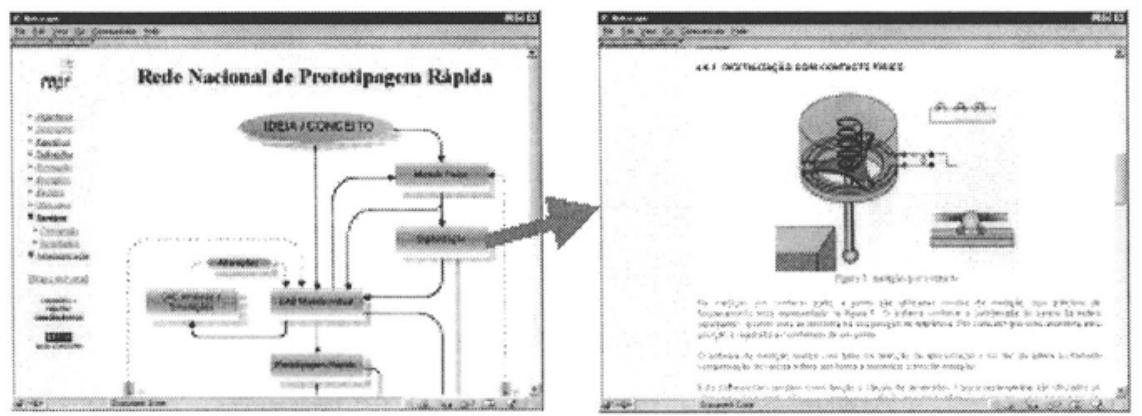

Figure 5 - The education service

\section{The Commerce Service}

A customer can submit a job description and digital representation of an object to be built, in order to get a budget for the production of the prototype. Presently the job description is then sent to all the members of the consortium for quotation. Quotations are then assembled by the Commerce Service and delivered to the customer. In the future, RNPR's DSS will be used to analyze the project and to submit it to one or more members of the consortium based on the technology (or melange of technologies) that would produce the desired result. The RNPR then will supply the outcome to the customer and, will assure both billing and payment (EDI).

\section{CONCLUSIONS AND FUTURE WORK}

To assist in information exchange among tools, UNINOVA developed a Web Portal, during RNPR. The portal enables a set of tools that allow the integration of several applications using standards. The goals were reached through the adaptation of 
existing applications selected in the market and the integration of product models, processes and resources in a global and standard information system.

The establishment of a DSS (Decision Support System) helped to fulfil two areas: primarily to assist in the design and development cycle of a project, and secondly, as a collateral effect, the collecting of data related to all projects, working in this sense as a data-warehousing tool.

During last year, the use of the Web portal made clear that two more features should be added to the platform in order to fulfil the project requirements: a comparison of technologies, and the commerce service. Given the natural competitiveness among each of the different technology holders and while the Portuguese market regarding the use of RP and RE is not stabilised, it was impossible to create the synergy to allow an completely "automated" computer program to direct customers to a given industrial RP facility. Nevertheless, we expect that with time, the features offered in a simplified process of electronic commerce service, for job submission and consortium quotation, will conduct RNPR's partners to request more sophisticated solutions.

\section{ACKNOWLEDGEMENTS}

The authors would like to thank without exception, to all the partners working in the RNPR projects' consortium and, in particular, we acknowledge the Portuguese National Commission that manages the PEDIP II program, for the financed budget and its support and trust in our ideas and developments.

\section{REFERENCES}

1. A. Luís Osório, Manuel M. Barata, Pierre Gison. Communication infrastructures requirements in a VE, Infrastructures for Virtual Enterprises. Networking Industrial Enterprises. Kluwer Academic Publishers. Edited by Luis M. Camarinha-Matos, Hamideh Afsarmanesh, IFIP. pages 65-77.1999

2. Antonio Osório. RNPR Advancement Project - National Network for Rapid Prototyping. Revista "O MOLDE”. Director Fernando Pedro. CEFAMOL, Legal Deposit Nº. 22499/99. pages 5-8, 1999

3. Boissier, R. et al. 1995. "Architecture solutions for integrating CAD, CAM and machining in small companies". In Camarinha-Matos and Hamideh-Afsarmanesh (eds). Proceedings of the IEEE / ECLA / IFIP International Conference on Architectures and Design Methods for Balanced Automation Systems (Chapman \& Hall, London), pages 407-416, 1995.

4. D. Dutta, N. Kikuchi, P. Papalmbros, F. Prinz, and L. Weiss. Project MAXWELL: Towards Rapid Realization of Superior Products. In H. L. Marcus, J. J. Beaman, J. W. Barlow, D. L. Bourell, and R. H. Crawford, editors, Solid Freeform Fabrication Symposium, pages 54-62. University of Texas at Austin, August 1992. Austin, Texas, USA.

5. ISO 14959, "ISO 14959 - Partl - Overview and Fundamentals Principles", Sep 97.

6. ISO TC184/SC4/WG7 N262, 1992, "ISO 10303 - Partl - Overview and Fundamentals Principles", 15 Sep 92.

7. R. J. Donahue and R. S. Turner. CAD Modelling and Alternative Methods of Information Transfer for Rapid Prototyping Systems. In National Conference on Rapid Prototyping, pages 221-235. University of Dayton and EMTEC, June 1991. Dayton, OH, USA.

\footnotetext{
i Other synonymous of the Rapid Prototyping (RP) term are Desktop Manufacturing (DM), Layered Manufacturing (LM), Solid Freeform Fabrication (SFF), and Solid Freeform Manufacturing (SFM).
} 